\title{
Crescimento da base craniana nos diferentes tipos faciais nos relacionamentos maxilomandibulares ortopédicos de Classe I, II e III. Parte 3 (Crescimento médio de CF-Po)
}

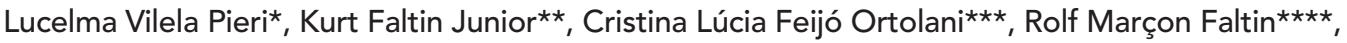

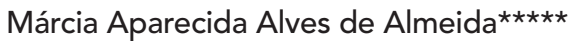

\section{Resumo}

Objetivo: este estudo retrospectivo avaliou o crescimento médio de CF-Po nos diferentes tipos faciais nos relacionamentos maxilomandibulares ortopédicos de Classe I, II e III. Metodologia: uma amostra aleatória de 300 pacientes brasileiros leucodermas (131 do gênero masculino, 169 do gênero feminino), com idade média inicial de 10 anos e 2 meses (dentadura mista) e final de 14 anos e 8 meses (segundos molares em oclusão) e tempo médio de observação de 4 anos e 5 meses, foi selecionada em uma clínica particular, em São Paulo, Brasil. Havia 118 pacientes Classe I, 151 Classe II e 31 Classe III. Todas as 600 radiografias cefalométricas laterais foram obtidas no mesmo aparelho de raios-x. As análises de Ricketts e Schwarz modificadas por Faltin foram usadas. A medida linear CF-Po foi feita manualmente pelo mesmo examinador em T1 e T2. Os relacionamentos foram estudados juntos e separadamente, considerando tipo facial e gênero. A análise de variância de ANOVA foi aplicada. Resultados e Conclusões: o tipo facial retrovertido mostrou-se significativamente desfavorável à correção de Classe III mandibular no gênero masculino; com tendência desfavorável à Classe II mandibular e crescimento ântero-posterior favorável na Classe I. O neutrovertido mostrou-se significativamente favorável à correção de Classe III mandibular em ambos os gêneros; com tendência favorável à correção de Classe II mandibular e ao crescimento ânteroposterior na Classe I. O provertido mostrou-se significativamente desfavorável à correção de Classe III em ambos os gêneros e favorável na Classe II mandibular e com tendência favorável de crescimento ântero-posterior na Classe I, principalmente no gênero masculino.

Palavras-chave: Base craniana. Crescimento. Tipos faciais. Relacionamento maxilomandibular ortopédico.

* Especialista em Ortodontia-Ortopedia Facial (APCD São José do Rio Preto). Mestre em Ortodontia (UNIP-SP).

** Especialista em Ortodontia-Ortopedia Facial. Pós-graduado e Doutor em Ortopedia Maxilar pela Universidade de Bonn, Alemanha. Professor Titular do Curso de Especialização e Mestrado em Ortodontia-Ortopedia Facial (UNIP-SP). Professor convidado do Departamento de Ortopedia Facial da Universidade de Ulm, Alemanha.

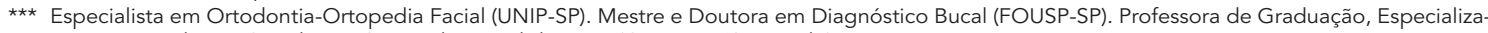
ção e Mestrado em Ortodontia-Ortopedia Facial da UNIP (Campinas/São Paulo).

**** Especialista em Ortodontia-Ortopedia Facial. Mestre em Biologia Celular e Tecidual pelo ICB/USP. Doutor em Ortopedia Facial pela Universidade de Ulm-Alemanha. Professor Adjunto do Departamento de Ortodontia-Ortopedia Facial (UNIP-SP).

***** Especialista e Mestranda em Ortodontia-Ortopedia Facial (UNIP-SP). Professora Assistente do Curso de Especialização em Ortodontia-Ortopedia Facial (UNIP-SP). 


\section{INTRODUÇÃO E REVISÃO DA LITERATURA}

O pório anatômico sofre um deslocamento posterior com o crescimento craniofacial, sendo que este pode ser medido cefalometricamente através da medida linear CF-Po, preconizada por Ricketts, Roth e Chaconas ${ }^{17}$ Esta medida dá o posicionamento das articulações temporomandibulares no sentido ântero-posterior (ATMs), conseqüentemente, o posicionamento ântero-posterior da mandíbula. Portanto, a medida linear CF-Po é de grande valor ao diagnóstico e o prognóstico individualizado para o tratamento de desarmonias dentofaciais, no paciente em crescimento.

Já em 1955, Ricketts ${ }^{14}$ relatou que a área da base craniana tem uma influência importante sobre o prognatismo facial total e no estabelecimento da relação ântero-posterior da maxila com a mandíbula.

Segundo Melsen ${ }^{10}$, Thilander e Ingervall ${ }^{20}$, através de estudos histológicos, e Nakamura et al. ${ }^{11,12}$, através de ressonância magnética, o fechamento completo da sincondrose esfenoccipital ocorre até os 17 anos de idade em indivíduos do gênero feminino e 20 anos nos do masculino. Para Krogman ${ }^{7}$, este ocorre no período entre a erupção dos segundos e terceiros molares permanentes. Para Melsen ${ }^{9}$, após a erupção de todos os caninos permanentes, pré-molares e segundos molares permanentes.

Segundo Ricketts ${ }^{15}$, a Classe II piora com a idade. Franchi et al. ${ }^{6}$ mostraram que mudanças maiores no crescimento mandibular, em correspondência de um estágio específico de maturação vertebral cervical (estágio 3 ao 4), representam o período mais favorável à correção de deficiências mandibulares, porque este inclui a porção ascendente da aceleração do crescimento da puberdade, com maior resposta biológica da cartilagem mandibular.

Sobre a situação do pório, Langlade ${ }^{8}$ mostrou que a medida linear CF-Po (centro da face - ponto obtido na interseção do plano vertical pterigóideo, PTV, com o plano de Frankfurt - ao pório anatômico na porção superior do meato acústico externo) tem a norma clínica de $39 \mathrm{~mm}$ aos 9 anos de idade, sendo que esse valor aumenta com o crescimento em torno de $0,5 \mathrm{~mm} / \mathrm{ano}$, mas é influenciado pelo tipo facial; tendo um desviopadrão de $\pm 2,2 \mathrm{~mm}$. A diminuição desta medida constitui um dos sinais de alarme da posição anterior das articulações temporomandibulares, observada freqüentemente no crescimento das prognatias mandibulares.

Enlow $^{3}$; Langlade ${ }^{8}$; Ngan et al. ${ }^{13}$; Silva et al. ${ }^{18}$; Bacetti, Franchi ${ }^{1}$; Cotrim-Ferreira ${ }^{2}$ e Singh et al. ${ }^{19}$ alertaram quanto ao prognóstico desfavorável à correção de prognatismo mandibular para o tipo braquifacial, no relacionamento maxilomandibular ortopédico de Classe III. Segundo Ricketts ${ }^{16}$, vários fatores devem ser vistos no prognóstico da Classe III, eles são: (1) corpo longo da mandíbula do ponto Xi ao Pm; (2) posição anterior do ponto $\mathrm{Xi}$; (3) pequena distância da vertical pterigóide ao pório; (4) base craniana anterior curta; (5) ângulo goníaco obtuso; (6) pequena altura do ramo e (7) ângulo obtuso da deflexão craniana.

Segundo Faltin Jr. ${ }^{4}$, no sentido vertical, a harmonia facial individual, respeitando os tipos faciais básicos de Ricketts, é realizada através da relação inversa existente entre o ângulo do eixo facial $\left(90 \pm 3^{\circ}\right)$ e os ângulos da altura facial total $\left(60 \pm 3^{\circ}\right)$ e a altura da dentadura $\left(45 \pm 3^{\circ}\right)$. A Ortodontia-Ortopedia Facial atual não aceita mais valores absolutos de normalidade ou padrões considerados normais, já que cada indivíduo apresenta uma arquitetura facial própria, sendo estes procedimentos especialmente necessários na população brasileira, na qual existe uma grande miscigenação racial. Ainda, segundo Faltin $\mathrm{Jr}^{4}{ }^{4}$, a harmonia facial é expressa por uma combinação de normas flutuantes de ângulos e proporções.

Este estudo retrospectivo teve como finalidade verificar o crescimento médio de CF-Po (posição das articulações temporomandibulares no sentido ântero-posterior), nos diferentes tipos faciais nos relacionamentos maxilomandibulares ortopédicos 


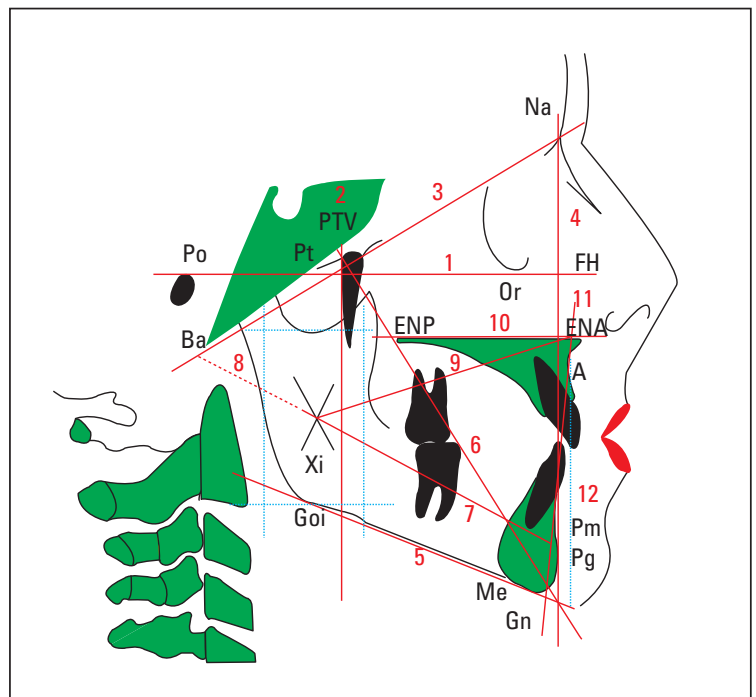

FIGURA 1 - Linhas e planos:

Análise de Ricketts modificada por Faltin:

1) Plano Horizontal de Frankfurt (FH);

2) Plano Pterigóideo Vertical (PTV):

3) Plano Ba-Na ou Plano da Base do Crânio;

4) Plano Facial (Na-Pg);

5) Plano Mandibular (Go-Me);

6) Eixo Facial (Pt-Gn);

7) Eixo do Corpo Mandibular (Xi-Pm);

8) Prolongamento de Xi-Pm;

9) Linha ENA - Xi;

Análise de Schwarz modificada por Faltin:

5) Plano Mandibular (Goi-Me):

10) Plano Palatino (ENA-ENP);

11) Linha A - Pm;

12) Linha $A-P m(D E V E)$

de Classe I, II e III, com a amostragem total e subdividida por gênero.

\section{MATERIAL E MÉTODOS}

A amostra de 300 pacientes brasileiros leucodermas (131 do gênero masculino, 169 do gênero feminino), com relacionamentos maxilomandibulares ortopédicos (118 Classe I, 151 Classe II e 31 Classe III), com idade média inicial de 10 anos e 2 meses e final de 14 anos e 8 meses, tempo médio de observação de 4 anos e 5 meses, os quais se apresentavam em $\mathrm{Tl}$ na dentadura mista, período visualizado na radiografia panorâmica, e em T2 na dentadura permanente com os segundos molares permanentes em oclusão, visualizado na telerradiografia em norma lateral obtida na posição de

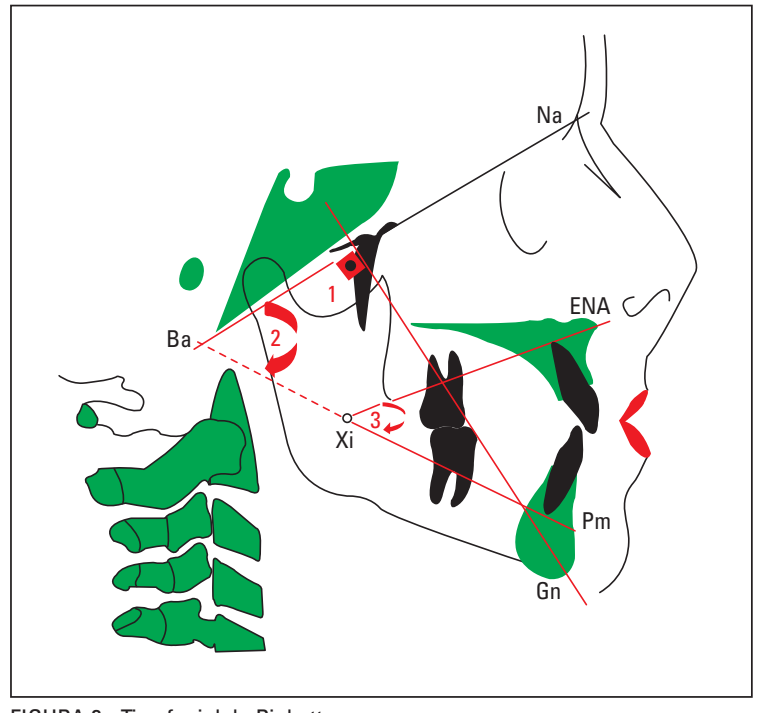

FIGURA 2 - Tipo facial de Ricketts:

1) Ângulo do Eixo Facial $\left(90 \pm 3^{\circ}\right)$;

2) Altura Facial Total $\left(60 \pm 3^{\circ}\right)$;

3) Altura da dentadura $\left(45 \pm 3^{\circ}\right)$.

máxima intercuspidação dentária, foi selecionada aleatoriamente de 1.400 pacientes que fazem parte do arquivo da clínica particular de um dos autores, na cidade de São Paulo-Brasil (Tab. 1).

Todas as 600 radiografias cefalométricas laterais foram obtidas a partir do mesmo aparelho de raios-x e com magnificação de 10\%. As análises cefalométricas de Ricketts ${ }^{17}$ (modificada por FAL$\mathrm{TIN}^{4}$ ) e Schwarz (modificada por FALTIN et al. ${ }^{5}$, através de suas linhas e planos (Fig. 1), determinaram os tipos faciais (Retrovertido, Neutrovertido e Provertido) (Fig. 2) e de relacionamentos maxilomandibulares ortopédicos no sentido ântero-posterior (Classe I, II e III) (Fig. 3), respectivamente.

A determinação dos tipos faciais e de relacionamentos maxilomandibulares ortopédicos, assim como da medida linear CF-Po (Fig. 4), foram feitas manualmente, pelo mesmo examinador, uma única vez, em T1 e T2. Depois, a medida linear CF-Po foi submetida a um tratamento estatístico. 


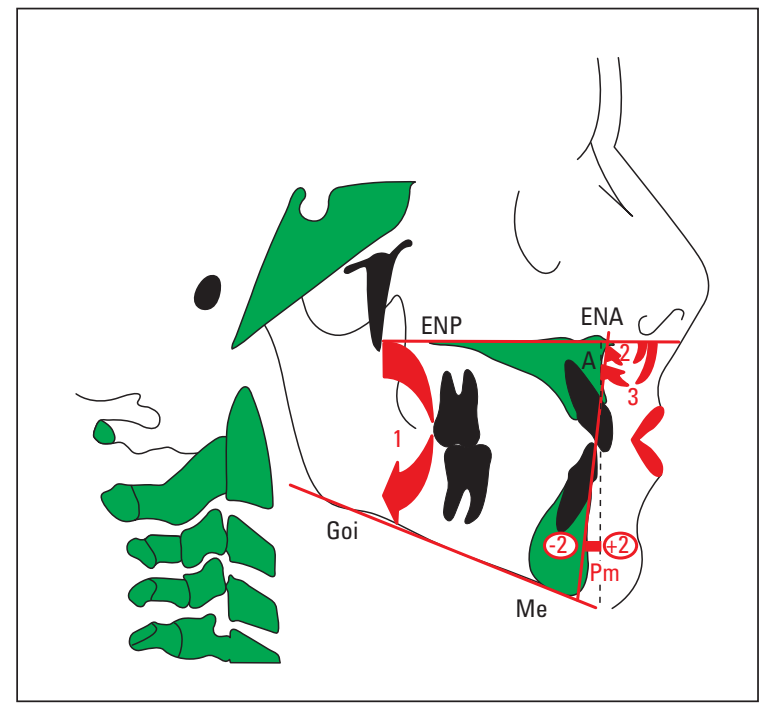

FIGURA 3 - Análise Schwarz modificada por Faltin.

1) Ângulo Basal $\left(20 \pm 10^{\circ}\right)$ :

2) Ângulo Pm-A-PI.Palatino $\left(90 \pm 7^{\circ}\right)$;

3) Ângulo DEVE (calculado).

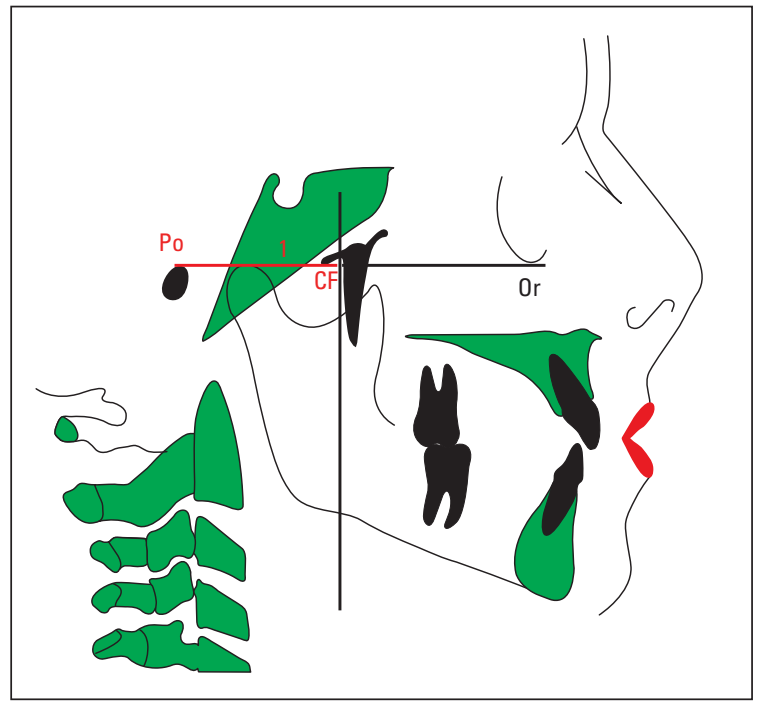

FIGURA 4 - Medidas lineares. 1) CF-Po.

Tabela 1 - Distribuição da amostra.

\begin{tabular}{|c|c|c|c|c|c|}
\hline \multirow{2}{*}{ gênero } & \multirow{2}{*}{ tipo facial } & \multicolumn{3}{|c|}{ Classe } & \multirow{2}{*}{ total } \\
\hline & & $\mathbf{I}$ & II & III & \\
\hline \multirow{3}{*}{ feminino } & retrovertido & 19 & 29 & 4 & 52 \\
\hline & neutrovertido & 34 & 35 & 3 & 72 \\
\hline & provertido & 17 & 25 & 3 & 45 \\
\hline \multirow{3}{*}{ masculino } & retrovertido & 16 & 18 & 7 & 41 \\
\hline & neutrovertido & 20 & 24 & 11 & 55 \\
\hline & provertido & 12 & 20 & 3 & 35 \\
\hline total & & 118 & 151 & 31 & 300 \\
\hline
\end{tabular}

\section{Análise estatística}

Nas análises seguintes, o teste de Levene foi utilizado para testar a homogeneidade das variâncias dos grupos.

Existiu homogeneidade quanto à idade média inicial e final dos grupos: gênero masculino (10 anos e 2 meses; 14 anos e 7 meses, respectivamente) e feminino (10 anos e 3 meses; 14 anos e 8 meses, respectivamente). O mesmo ocorreu com o tempo médio de observação, que foi de 4 anos e 4 meses para o gênero masculino e de 4 anos e 7 meses para o feminino.
Depois, utilizou-se a análise de variância de ANOVA para verificar se existia diferença significativa entre as médias (crescimento médio) de CF-Po. As comparações múltiplas pelo método de Tukey foram usadas para verificar se existia diferença significativa entre os tipos faciais e gênero.

\section{RESULTADOS}

Análise dos relacionamentos maxilomandibulares ortopédicos juntos (Classe I, II e III) com a amostragem subdividida em tipos faciais

Para CF-Po, o nível descritivo foi de 0,239 , por- 
tanto, não existiu diferença significativa entre as variabilidades (desvios-padrão) dos diversos grupos.

Existiu interação dos fatores Classe e Tipo Facial $(p=0,021)$. Porém, não existiram diferenças significativas entre os tipos faciais $(p=0,174)$ para CF-Po. Embora, no relacionamento maxilomandibular ortopédico de Classe III, a média do grupo Classe III/provertido $(-0,3 \pm 0,4 \mathrm{~mm})$ foi maior ( $\mathrm{p}=0,0615)$ que a do grupo Classe III/ neutrovertido $(-2,5 \pm 1,3 \mathrm{~mm})$, esta diferença não foi significativa.

\section{Análise por Classe de relacionamento maxi- lomandibular ortopédico com a amostragem subdividida em tipos faciais}

Para CF-Po, o nível descritivo para os grupos Classe I, II e III foi 0,783; 0,385 e 0,160, respectivamente. Portanto, não existiu diferença significativa entre a variabilidade (desvios-padrão) dos diversos grupos.

Tabela 2 - Comparações múltiplas pelo método de Tukey (CF-Po, Classe III / tipos faciais).

\begin{tabular}{cccc}
\hline tipo facial & retro & neutro & pro \\
\hline retro & & 0,3508 & 0,0818 \\
neutro & 0,3508 & & $0,0045^{*}$ \\
pro & 0,0818 & $0,0045^{*}$ & \\
\hline
\end{tabular}

* valores estatisticamente significantes.

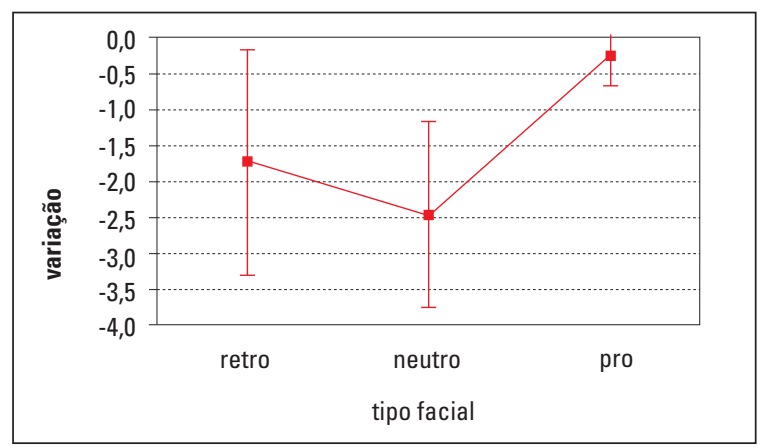

GRÁFICO 1 - Médias \pm 1 desvio-padrão para CF-Po (Classe III - tipos faciais).
Não existiu diferença significativa entre as médias dos tipos faciais para CF-Po nos relacionamentos maxilomandibulares ortopédicos de Classe I $(p=0,361)$ e II $(p=0,627)$. Porém, no relacionamento maxilomandibular de Classe III, a média do grupo provertido $(-0,3 \pm 0,4 \mathrm{~mm})$ foi significativamente menor no sentido negativo do sinal, ao nível descritivo de $0,006(\mathrm{p}<0,05)$, que a do grupo neutrovertido $(-2,5 \pm 1,3 \mathrm{~mm})$ (Tab. 2, Gráf. 1).

\section{Análise por Classe de relacionamento maxilo- mandibular ortopédico correlacionando gêne- ro e tipo facial}

Para CF-Po, o nível descritivo nos relacionamentos maxilomandibulares ortopédicos de Classe I, II e III foi de 0,831; 0,907 e 0,502, respectivamente. Portanto, as variabilidades (desviospadrão) dos grupos foram homogêneas, ou seja, a diferença entre a média dos dois gêneros foi igual nos três tipos faciais.

Tabela 3 - Comparações múltiplas pelo método de Tukey (CF-Po, Classe III, tipos faciais / gêneros).

\begin{tabular}{cccc}
\hline tipo facial & retro & neutro & pro \\
\hline retro & & 0,8836 & $0,0286^{*}$ \\
neutro & 0,8836 & & $0,0122^{*}$ \\
pro & $0,0286^{*}$ & $0,0122^{*}$ & \\
\hline * valores estatisticamente significantes. &
\end{tabular}

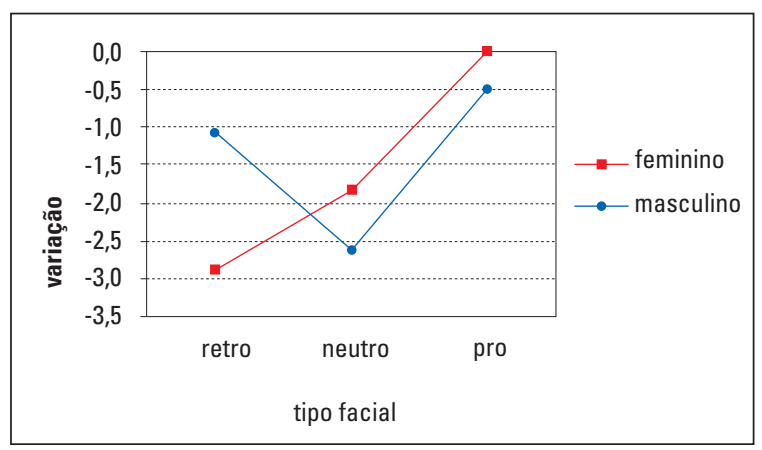

GRÁFICO 2 - Médias para CF-Po (Classe III - tipos faciais / gêneros). 
Existiu diferença significativa entre os tipos faciais somente no relacionamento maxilomandibular ortopédico de Classe III, neste a média do grupo provertido $(-0,3 \pm 0,4 \mathrm{~mm})$ foi significativamente menor no sentido negativo do sinal ao nível descritivo de 0,0122 e $0,0286, p<0,05$, que a do grupo neutrovertido $(-2,5 \pm 1,3 \mathrm{~mm})$ e retrovertido $(-1,7 \pm 1,6 \mathrm{~mm})$, respectivamente (Tab. 3, Gráf. 2).

Não existiu interação significativa entre os fatores gênero e tipo facial nos relacionamentos maxilomandibulares ortopédicos de Classe I ( $\mathrm{p}=$ $0,410)$, II ( $p=0,421)$ e III $(p=0,057)$.

Existiu diferença significativa entre os gêneros somente nos relacionamentos maxilomandibulares ortopédicos de Classe I e II, nestes, as médias para o gênero masculino $(-2,2 \pm 1,5 \mathrm{~mm}$;

Tabela 4 - Médias e desvios-padrão para CF-Po em cada tipo facial nos relacionamentos maxilomandibulares ortopédicos de Classes I, II e III.

\begin{tabular}{ccc}
\hline tipo facial & Classe & $\begin{array}{c}\text { médias e desvios-padrão } \\
\text { CF-Po }(\mathbf{m m})\end{array}$ \\
\hline \multirow{2}{*}{ retrovertido } & I & $-1,8(1,3)$ \\
& II & $-2,1(1,5)$ \\
neutrovertido & III & $-1,7(1,6)$ \\
\hline \multirow{2}{*}{ provertido } & I & $-1,7(1,4)$ \\
& II & $-1,8(1,5)$ \\
& III & $-2,5(1,3)$ \\
\hline & I & $-2,1(1,6)$ \\
& II & $-2,0(1,7)$ \\
& III & $-0,3(0,4)$ \\
\hline
\end{tabular}

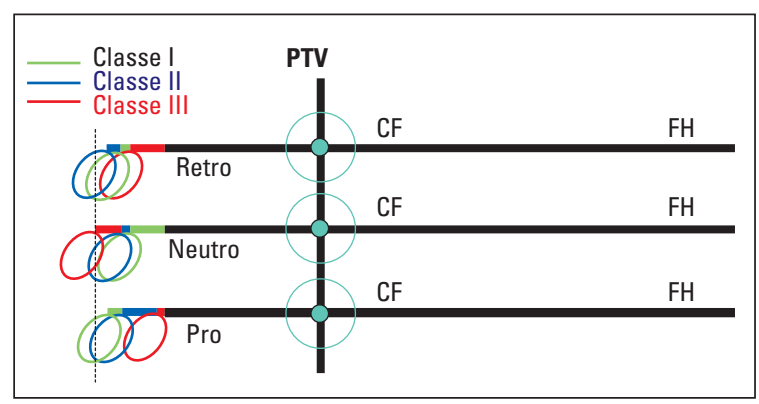

FIGURA 5 - Superposições do crescimento médio de CF-Po no plano horizontal de Frankfurt (CF) Classes em cada tipo facial.
$-2,4 \pm 1,7 \mathrm{~mm}$ ) foram significativamente maiores, no sentido negativo do sinal, do que as para o feminino $(-1,6 \pm 1,4 \mathrm{~mm} ;-1,6 \pm 1,5 \mathrm{~mm})$ ao nível descritivo de 0,002 e 0,039 , p < 0,05, respectivamente. $\mathrm{Na}$ Classe III ( $\mathrm{p}=0,739)$, não existiu diferença significativa entre os gêneros.

O crescimento médio geral (T2-T1) de CF-Po pode ser observado nas superposições dos relacionamentos maxilomandibulares ortopédicos de Classe I, II e III em cada tipo facial sobre o Plano Horizontal de Frankfurt (FH) em CF (Fig. 5). Também, considerando o gênero (Fig. 6). As médias e desvios-padrão para CF-Po em cada tipo facial nos relacionamentos maxilomandibulares ortopédicos de Classe I, II e III encontram-se nas tabelas 4 e 5 .

Tabela 5 - Médias e desvios-padrão para CF-Po em cada tipo facial (Gênero) nos relacionamentos maxilomandibulares ortopédicos de Classes I, II e III.

\begin{tabular}{cccc}
\hline \multirow{2}{*}{ tipo facial } & Classe & \multicolumn{2}{c}{$\begin{array}{c}\text { médias e desvios-padrão } \\
\text { CF-Po (mm) }\end{array}$} \\
\cline { 3 - 4 } & & feminino & masculino \\
\hline \multirow{2}{*}{ retrovertido } & I & $-1,3(1,0)$ & $-2,4(1,4)$ \\
& II & $-1,8(1,3)$ & $-2,7(1,5)$ \\
& III & $-2,9(1,1)$ & $-1,1(1,1)$ \\
\hline \multirow{2}{*}{ neutrovertido } & I & $-1,5(1,3$ & $-1,9(1,6)$ \\
& II & $-1,8(1,5)$ & $-1,9(1,6)$ \\
\hline \multirow{2}{*}{ provertido } & III & $-1,8(1,0)$ & $-2,6(1,3)$ \\
\hline & I & $-2,0(1,7)$ & $-2,3(1,5)$ \\
& II & $-1,3(1,4)$ & $-2,8(1,8)$ \\
& III & $-0,0(0,0)$ & $-0,5(0,5)$ \\
\hline
\end{tabular}

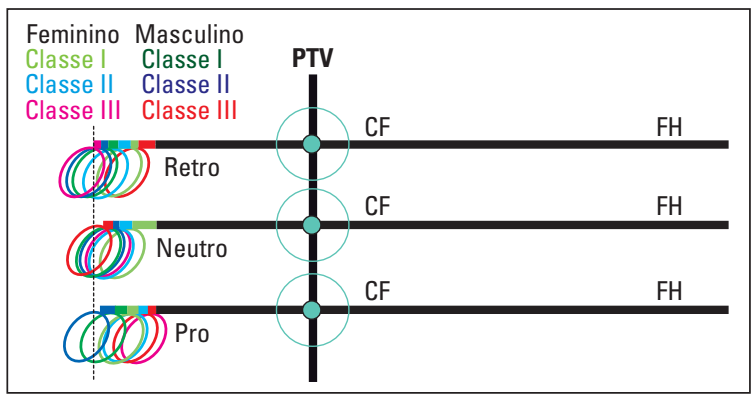

FIGURA 6 - Superposições do crescimento médio de CF-Po no plano horizontal de Frankfurt (CF) Classes em cada tipo facial (Gênero). 
$\mathrm{O}$ crescimento médio esperado para CF-Po (médias de crescimento esperadas) no tempo médio de observação de 4 anos e 5 meses, baseado no crescimento médio anual (média de variação anual) para os 300 pacientes foi o seguinte: CFPo (total -2,1 mm); gênero masculino $(-2,6 \mathrm{~mm})$ e feminino $(-1,7 \mathrm{~mm})$. Estas médias de crescimento esperado podem ser comparadas aos valores medidos (Tab. 4, 5).

\section{DISCUSSÃO}

A idade mínima em $\mathrm{Tl}$ foi de 7,1 anos para os dois gêneros. Na segunda observação desta pesquisa, T2, a idade média final dos pacientes foi de 14 anos e 8 meses, com idade máxima de 17,8 e 17,9 anos para os gêneros feminino e masculino, respectivamente, com os segundos molares permanentes em oclusão. $\mathrm{Na}$ segunda observação, segundo Melsen ${ }^{10}$; Thilander, Ingervall ${ }^{20}$; Nakamura et al. ${ }^{11,12}$; Krogman ${ }^{7}$ e Melsen ${ }^{9}$, o crescimento craniofacial destes pacientes, na sua maior parte, já tinha ocorrido.

Esta pesquisa investigou o crescimento médio de CF-Po nos diferentes tipos faciais nos relacionamentos maxilomandibulares ortopédicos de Classe I, II e III. Segundo Langlade ${ }^{8}$, esta medida tem uma norma clínica de $-39 \pm 2,2 \mathrm{~mm}$ aos 9 anos de idade, tendo um incremento anual de $-0,5 \mathrm{~mm}$. Segundo Ricketts et al. ${ }^{17},-38,5 \pm 2 \mathrm{~mm}$ aos 8,5 anos de idade e incremento anual de $-0,5 \mathrm{~mm}$. Para a análise cefalométrica, foi estabelecida a adição de incrementos anuais na norma clínica a partir dos 8,5 anos até a idade de 15 anos, para ambos os gêneros.

\section{Análise dos relacionamentos maxilomandibu- lares ortopédicos juntos (Classe I, II e III) com a amostragem subdividida em tipos faciais \\ Não existiu diferença significativa entre os tipos de relacionamentos maxilomandibulares ortopédicos e nem entre os tipos faciais para CF-Po, quando todos os dados foram analisados juntos.}

\section{Análise por Classe de relacionamento maxi- lomandibular ortopédico com a amostragem subdividida em tipos faciais}

O tipo facial provertido no relacionamento maxilomandibular ortopédico de Classe III mostrou-se significativamente desfavorável à correção do relacionamento maxilomandibular ortopédico de Classe III mandibular, o contrário ocorreu para o neutrovertido. Segundo Langla$\mathrm{de}^{8}$, a diminuição desta medida constitui um dos sinais de alarme da posição anterior das articulações temporomandibulares, que freqüentemente é observada no crescimento das prognatias mandibulares. Nesta pesquisa, esta medida estava significativamente diminuída no provertido e dentro do esperado no neutrovertido.

\section{Análise por Classe de relacionamento maxilo- mandibular ortopédico correlacionando gêne- ro e tipo facial}

O tipo facial retrovertido mostrou um prognóstico significativamente desfavorável à correção de prognatismo mandibular. Nos pacientes que já possuem a altura ântero-inferior aumentada, a cirurgia ortognática provavelmente se faz necessária após o encerramento do crescimento craniofacial. Embora, não tenha existido interação dos fatores gênero e tipo facial nos relacionamentos maxilomandibulares ortopédicos de Classe I, II e III, nos pacientes masculinos/retrovertidos do grupo Classe III (22,6\% da amostra) a medida linear CF-Po estava diminuída, mostrando tendência desfavorável à correção do relacionamento maxilomandibular ortopédico de Classe III no gênero masculino, o que não ocorreu no feminino. Já no relacionamento ortopédico de Classe II esta medida estava levemente aumentada, mostrando uma tendência desfavorável leve à sua correção. No de Classe I estava levemente diminuída em ambos os gêneros, ou seja, com boa tendência de crescimento ântero-posterior da mandíbula.

Segundo Silva et al. ${ }^{18}$, a Classe III verdadeira é uma deformidade multifatorial em sua etiolo- 
gia, podendo sofrer inúmeras influências intrínsecas ou extrínsecas, na qual fatores genéticos ou ambientais exerçam influência, de forma distinta, em diferentes períodos. Ainda, afirmou que a base craniana, por ser uma região inacessível aos efeitos terapêuticos da mecânica ortodôntica e ortopédica, possui uma grande determinação genética e um término precoce do crescimento e desenvolvimento em relação às outras regiões faciais. Por isso, deve ser considerada como uma importante fonte de informações para o diagnóstico, prognóstico e planejamento dos casos, com uma individualização para cada caso, baseada no conhecimento sobre o crescimento e o desenvolvimento craniofacial, pois as análises cefalométricas aplicadas de forma matemática podem mascarar a real condição esquelética, exigindo do profissional conhecimento sobre a atividade das sincondroses da base do crânio na previsão da influência da base craniana sobre o complexo craniofacial.

O tipo facial neutrovertido mostrou-se significativamente favorável à correção do relacionamento Classe III em ambos os gêneros, tendo um deslocamento normal do pório com o crescimento craniofacial. O grupo Classe III neutrovertido constituiu-se de 14 pacientes (45\% da amostra) o que favoreceu a correção de praticamente metade dos casos com prognatismo mandibular. No relacionamento de Classe II, mostrou-se significativamente favorável à sua correção, principalmente no gênero masculino, que apresentou esta medida diminuída. No relacionamento de Classe I, principalmente no gênero masculino, esta medida também apresentou-se diminuída, demonstrando maior tendência de crescimento ânteroposterior da mandíbula neste gênero.

O tipo facial provertido mostrou-se significativamente desfavorável à correção do relacionamento maxilomandibular ortopédico de Classe III mandibular em ambos os gêneros. A medida CF-Po estava significativamente diminuída no provertido. Isto é pior para indivíduos do gênero masculino, que crescem mais e por um período de tempo maior. Estes dados estão em concordância com Enlow ${ }^{3}$; Langlade ${ }^{8}$; Ngan et al. ${ }^{13}$; Silva et al. ${ }^{18}$; Bacetti, Franchi ${ }^{1}$; Cotrim-Ferreira ${ }^{2}$ e Singh et al. ${ }^{19}$, quanto ao prognóstico desfavorável à correção de prognatismo mandibular para o tipo facial provertido, no relacionamento maxilomandibular ortopédico de Classe III. Segundo Enlow $^{3}$, os japoneses, sendo na maioria braquicefálicos e provavelmente com tipo facial provertido, têm tendência maior para más oclusões de Classe III e perfil prognata.

O grupo de relacionamento maxilomandibular ortopédico de Classe III masculino/provertido mostrou tendência desfavorável duas vezes, pelo gênero e pelo tipo facial, portanto, concordando com Ricketts ${ }^{16}$; Enlow ${ }^{3}$; Langlade ${ }^{8}$; Ngan et al. ${ }^{13}$; Bacetti e Franchi ${ }^{1}$, quanto ao sinal de alarme das prognatias mandibulares. $\mathrm{O}$ grupo Classe III feminino/provertido mostrou tendência mais desfavorável que o grupo masculino/provertido, porém, sendo mais preocupante para o gênero masculino, uma vez que este tende a crescer mais por um período de tempo maior. Portanto, segundo Ricketts ${ }^{16}$, vários fatores devem ser vistos no prognóstico da Classe III, eles são: (1) corpo longo da mandíbula, do ponto $\mathrm{Xi}$ ao $\mathrm{Pm}$; (2) posição anterior do ponto $\mathrm{Xi}$, (3) pequena distância da vertical pterigóide ao pório; (4) base craniana anterior curta; (5) ângulo goníaco obtuso; (6) altura pequena do ramo e (7) ângulo obtuso da deflexão craniana.

O provertido ainda foi significativamente favorável à correção de Classe II mandibular em ambos os gêneros. No relacionamento maxilomandibular de Classe I, mostrou tendência favorável de crescimento ântero-posterior, principalmente no gênero masculino.

Somente nos relacionamentos maxilomandibulares ortopédicos de Classe I e II existiu diferença significativa entre os gêneros para CF-Po. $\mathrm{O}$ gênero masculino obteve médias maiores, no sentido negativo do sinal, que o feminino. Nesta pesquisa, houve maior predominância do relacio- 
namento maxilomandibular ortopédico de Classe III no gênero masculino (21 pacientes) que no feminino (10 pacientes), sendo que o crescimento médio foi significativamente maior para o masculino. Portanto, mostrou tendência desfavorável à correção do relacionamento maxilomandibular ortopédico de Classe III mandibular, principalmente para o gênero masculino.

\section{CONCLUSÕES}

1) O tipo facial retrovertido no relacionamento maxilomandibular ortopédico de Classe III mostrou-se significativamente desfavorável à correção de Classe III mandibular no gênero masculino e com tendência favorável no feminino. No de Classe II, tendência desfavorável à sua correção em ambos os gêneros. No de Classe I, tendência favorável de crescimento ântero-posterior.

2) O tipo facial neutrovertido no relacionamento maxilomandibular ortopédico de Classe III mostrou-se significativamente favorável à correção de Classe III mandibular em ambos os gê- neros. No de Classe II, tendência favorável à correção da Classe II mandibular, principalmente no gênero masculino. No de Classe I, tendência de crescimento ântero-posterior favorável, principalmente no gênero masculino.

3) O tipo facial provertido no relacionamento maxilomandibular ortopédico de Classe III mostrou-se significativamente desfavorável à correção de Classe III mandibular em ambos os gêneros. No de Classe II, significativamente favorável à correção da Classe II mandibular em ambos os gêneros. No de Classe I, tendência de crescimento ântero-posterior favorável, principalmente no gênero masculino.

Cranial base growth in different facial types in Class I, II and III orthopedic maxillomandibular relationship. Part 3 (Mean growth of CF-Po)

\begin{abstract}
Aim: This retrospective study assessed the cranial base growth and development in different facial types in Class I, II and III orthopedic maxillomandibular relationship. Methods: A random sample of 300 Brazilian Caucasian patients (131 males, 169 females), with initial mean age of 10 years and 2 months (mixed dentition) and final mean age 14 years and 8 months (second molar in occlusion) and mean observation time of 4 years and 5 months, was selected at a private clinic in São Paulo. There were 118 Class I; 151 Class II and 31 Class III. All 600 lateral cephalometric radiographs were taken using the same x-rays equipment. Ricketts and Schwarz analyses modified by Faltin were used. The same examiner performed manually CF-Po linear measure in T1 and T2. All Classes were studied together and separately, considering facial type and gender. ANOVA variance analysis was carried out. Results and Conclusions: Retroversion facial type was significantly unfavorable to Class III correction in males; it showed unfavorable tendency to mandibular Class II correction and favorable anteroposterior growth tendency in Class I. Neutroversion was significantly favorable to mandibular Class III correction in both genders; it showed favorable tendency to mandibular Class II correction and favorable anteroposterior growth tendency to Class I. Proversion was significantly unfavorable and favorable to Class III and Class II correction in both genders, respectively; it showed favorable anteroposterior growth tendency to Class I mainly in males.
\end{abstract}

Key words: Cranial base. Growth. Facial types. Orthopedic maxillomandibular relationship. 


\section{REFERÊNCIAS}

1. BACETTI, T.; FRANCHI, L. Shape-coordenate analysis of mandibular growth in untreated Class III malocclusions. In: CONGRESS OF THE EUROPEAN ORTHODONTIC SOCIETY/EOS, 74th CONGRESS OF THE GERMAN ORTHODONTIC SOCIETY/ DGKFO, 71th., 1998, Mainz. Abstracts... Mainz, 1998. p. 328.

2. COTRIM-FERREIRA, F. A. Estudo cefalométrico radiográfico longitudinal de algumas medidas angulares e lineares da base craniana, dos tipos morfológicos faciais e suas possíveis correlações. 1999. Tese (Doutorado em Diagnóstico Bucal)-Faculdade de Odontologia da Universidade de São Paulo, São Paulo, 1999.

3. ENLOW, D. Crescimento craniofacial. 3. ed. São Paulo: Artes Médicas, 1993.

4. FALTIN JR., K. A individualização do diagnóstico e conseqüentes opções de tratamento. In: GRUPO BRASILEIRO DE PROFESSORES DE ORTODONTIA E ODONTOPEDIATRIA, 1997, São Paulo. $7^{\circ}$ livro... São Paulo: [S.n.], 1997. p. 166-172.

5. FALTIN JR., K.; MACHADO, C. R.; REBECCHI, M. C. V. C. Valores médios da análise cefalométrica de Schwarz-Faltin para jovens brasileiros, leucodermas com oclusão normal. Rev. Soc. Paran. Ortodon., Curitiba, v. 1, n. 3, p. 31-42, nov./fev. 1997.

6. FRANCHI, L.; BACCETTI, T.; McNAMARA JR., J. A . Thin-plate spline analysis of mandibular growth. Angle Orthod., Appleton, v. 71, no. 2, p. 83-92, 2001.

7. KROGMAN, W. M. Studies in growth changes in the skull and face of anthropoids. Am. J. Anat., New York, v. 46, p. 315-353, 1930.

8. LANGLADE, M. Diagnóstico ortodôntico. 1. ed. São Paulo: Ed. Santos, 1995

9. MELSEN, B. Time of closure of the spheno-occipital synchondrosis determined on dry skulls: a radiographic craniometric study. Acta Odontol. Scand., Stockholm, v. 27, p. 73-90, 1969.

10. MELSEN, B. Time and mode of closure of the spheno-occipital synchondrosis determined on human autopsy material. Acta Anat., Basel, v. 83, p. 112-118, 1972.
11. NAKAMURA, Y.; KAWASAKI, K.; SEKYA, T.; KUWAHARA, Y.; KOBAYASHI, K. Observation of human spheno-occipital synchondrosis with magnetic resonance imaging. J. Japan Orthod. Soc., Tokyo, v. 55, no. 4, p. 282-286, 1996.

12. NAKAMURA, Y.; NODA, K.; KUWAHARA, Y.; MINYEONG, L.; TANAKA, S.; KAWASAKI, K.; KOBAYASHI, K. Magnetic resonance images and histology of the spheno-occipital synchondrosis in young Monkeys (Macaca Fuscata). Am. J. Orthod. Dentofacial Orthop., St. Louis, v. 155, no. 2, p. 138-142, 1999.

13. NGAN, P.; HÄGG, U.; YIU, C.; MERWIN, D.; WEY, S. H. Y. Cephalometric comparisons of chinese and caucasian surgical Class III Patients. Int. J. Adult. Orthodon. Orthognath Surg. Chicago, v. 12, no. 2, p.177-188, 1997

14. RICKETTS, R. M. Facial and denture changes during orthodontic treatment as analyzed from the temporomandibular joint. Am. J. Orthod., St. Louis, v. 41, p. 163-179, 1955.

$15 . \quad$ Dr. Robert M. Ricketts on growth prediction. Part 3. J. Clin. Orthod., Boulder, p. 420-434, July 1975.

$16 . \quad$ Dr. Robert M. Ricketts on early treatment. Part 3. J. Clin. Orthod., Boulder, p. 180-199, Mar. 1979.

17. RICKETTS, R. M.; ROTH, R. H.; CHACONAS, S. J. Orthodontic diagnosis and planning. 1. ed. [S.I.]: Rock Mountain, 1982.

18. SILVA, A. A.; CARVALHO, D. S.; ADIMARI, M. R. W. Previsão de crescimento: a relação entre a base do crânio e a Classe III de Angle. Rev. Dental Press Ortodon. Ortop. Maxilar, Maringá, v. 2, n. 4, p. 71-79, jul./ago. 1997.

19. SINGH, G. D.; McNAMARA JR., J. A.; LOZANOFF, S. Allometry of cranial base in pubertal korean subjects with Class III malocclusions: finite element morphometry. Angle Orthod., Appleton, v. 69, no. 6, p. 507-514, 1999.

20. THILANDER, B.; INGERVALL, B. The human spheno-occipital synchondrosis. II. A histological and microradiographic study of its growth. Acta Scand., Stockholm, v. 31, no. 5, p. 323-334, 1973.
Endereço de correspondência

Lucelma Vilela Pieri

Rua Franklin Martins, 1643

CEP:14.400-210 - Franca / SP

E-mail: lucelmapieri@ortodontista.com.br 